\title{
Formation and evolution of black hole and accretion disk in collapse of massive stellar cores
}

\author{
Yuichiro Sekiguchi \\ Yukawa Institute for Theoretical Physics, Kyoto University, Kyoto 606-8502, Japan \\ email: sekig@yukawa.kyoto-u.ac.jp
}

\begin{abstract}
We describe the results of our numerical simulations of the collapse of a massive stellar core to a $\mathrm{BH}$, performed in the framework of full general relativity incorporating finitetemperature equation of state and neutrino cooling. We adopt a $100 M_{\odot}$ presupernova model calculated by Umeda \& Nomoto (2008), which has a massive core with a high value of entropy per baryon. Changing the degree of rotation for the initial condition, we clarify the dependence of the outcome on this. When the rotation is rapid enough, the shock wave formed at the core bounce is deformed to be a torus-like shape. Then, the infalling matter is accumulated in the central region due to the oblique shock at the torus surface, hitting the hypermassive neutron star (HMNS) and dissipating the kinetic energy there. As a result, outflows can be launched. The HMNS eventually collapses to a $\mathrm{BH}$ and an accretion torus is formed around it. We also found that the evolution of the $\mathrm{BH}$ and torus depends strongly on the rotation initially given.
\end{abstract}

Keywords. Stellar core collapse, black hole, accretion disk, gamma-ray burst

\section{Introduction}

The observational associations (for a review, see Woosley \& Bloom (2006)) between LGRBs and supernovae has provided strong support to the so-called collapsar model (Woosley (1993); MacFadyen \& Woosley (1999)). In the collapsar model, a central core of a massive star is required to be rotating rapidly enough that a massive accretion disk can be formed around a BH. The observational association of LGRBs with Type Ic (and b) SNe and the requirement that the relativistic jets have to reach the stellar surface (Zhang \& Woosley (2004)) raise the serious problem that according to stellar evolution calculations, it is very difficult to produce pre-collapse cores which satisfy both the requirement of the collapsar model and the association of Type $\mathrm{Ib} / \mathrm{c}$ SNe, if magnetic torques and standard mass-loss rates are taken into account (Woosley \& Heger (2006)).

To resolve the above dilemma, several models have been proposed (see Fryer et al. (2007) for a review). All of the proposed progenitor models of LGRBs are anomalous in the sense that they are different from the progenitors of ordinary SNe (see Sekiguchi \& Shibata (2011) for a discussion). Qualitatively speaking, LGRB progenitor cores may be modeled by a rapidly rotating, higher-entropy core, regardless of their formation processes. Based on this assumption, we performed simulations of a massive stellar core with higher values of entropy collapsing to a $\mathrm{BH}$.

\section{Setting}

As a representative model of a high entropy core, we adopt a presupernova core of $100 M_{\odot}$ model calculated by Umeda \& Nomoto (2008) (hereafter denoted by UN100). The model has an iron core of large mass $M_{\text {core }} \approx 3.2 M_{\odot}$ and the central value of entropy per baryon is $s \approx 4 k_{B}$, which is much larger than that of an ordinary presupernova 


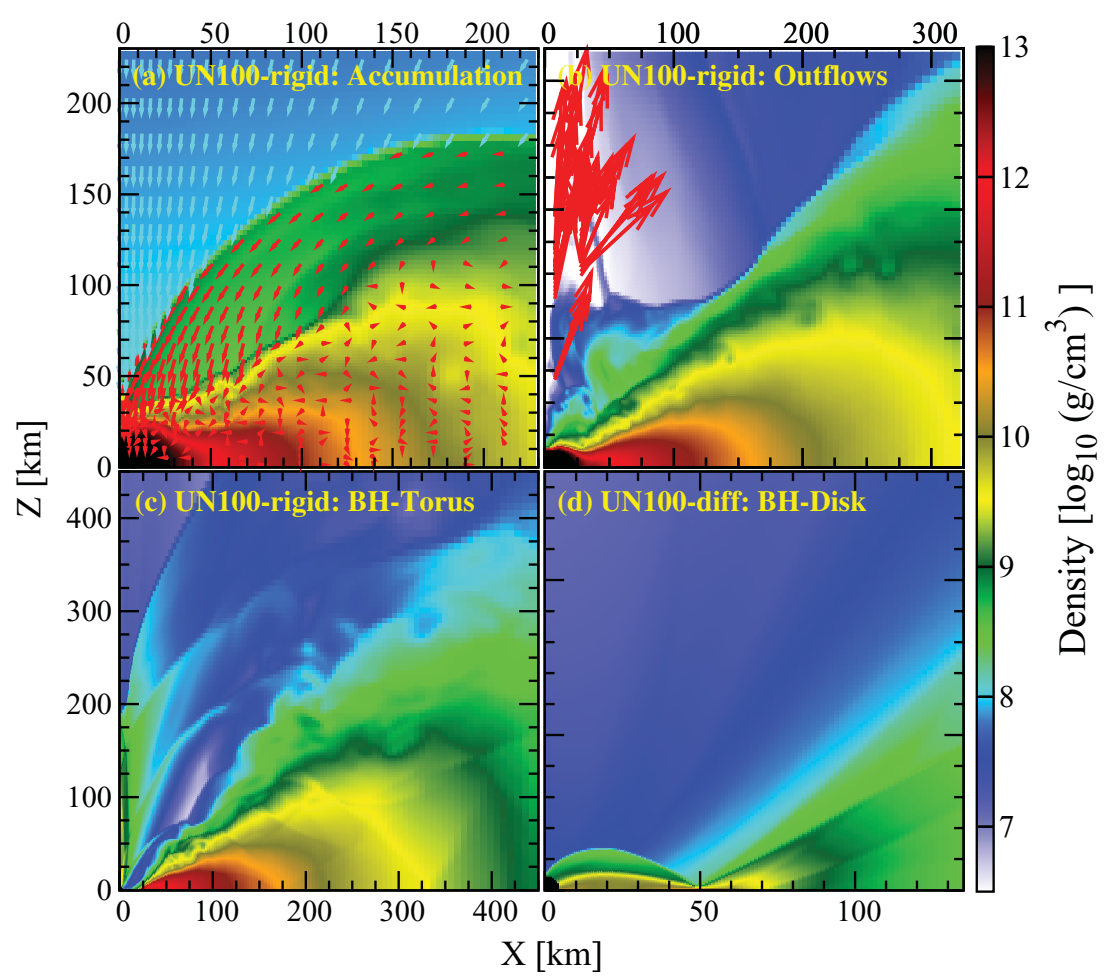

Figure 1. Contours of the rest-mass density in the $x$ - $z$ plane for UN100-rigid at (a) $t=645 \mathrm{~ms}$ (the mass accumulation onto the HMNS), (b) $1256 \mathrm{~ms}$ (the launch of outflows), and (c) 2225 ms (time-variable BH-Torus), and (d) for UN100-diff at $t=1150 \mathrm{~ms}$ (quiet BH-Disk).

core for which $s \lesssim 1 k_{B}$. Because the model UN100 is non-rotating, we add rotational profiles according to, $\Omega(\varpi)=\Omega_{0} \frac{R_{0}^{2}}{R_{0}^{2}+\varpi^{2}} \mathcal{F}_{\text {cut }}$, where $\varpi=\sqrt{x^{2}+y^{2}}$. We fix the central angular velocity as $\Omega_{0}=1.2 \mathrm{rad} / \mathrm{s}$ and consider two values of $R_{0}$; a rigid rotation model $\left(R_{0}=\infty\right.$, referred to as UN100-rigid) and a differential rotation $\left(R_{0}=R_{\text {core }}\right.$, referred to as UN100-diff) model $\dagger$. The cut-off factor $\mathcal{F}_{\text {cut }}$ is introduced so that the matter in the outer region would not escape from the computational domain.

The simulations are performed using a full GR code recently developed, Sekiguchi (2011). We assume axial and equatorial symmetries of the spacetime and the so-called Cartoon method (e.g., Shibata (2003)) is adopted. In numerical simulations, we adopt a nonuniform grid, in which the grid spacing is increased according to the rule $d x_{j+1}=$ $(1+\delta) d x_{j}$, where $\delta$ is a constant. In addition, a regridding technique (e.g., Sekiguchi \& Shibata (2005)) is adopted. We set an infalling boundary condition at the outer boundary.

\section{Results}

The dynamics of the collapse in UN100-rigid and UN100-diff is similar to that in the the ordinary SN simulations, until the shock wave formed in the core bounce stalls. However, the dynamics of UN100-diff and UN100-rigid (and the ordinary SN) in the later phase is qualitatively different as follows. Figure 1 shows contour plots of the restmass density in the $x-z$ plane at selected time slices for UN100-diff (d) and UN100-rigid (a)-(c). (i) Due to the faster rotation of the outer region in UN100-rigid, the shock wave is deformed to be a torus-like configuration (see Fig. 1(a)), which is one of the

$\dagger$ We note that $\Omega_{0}$ is much smaller than the Kepler value $\left(M_{\text {core }} / R_{\text {core }}^{3}\right)^{1 / 2} \approx 5.2 \mathrm{rad} / \mathrm{s}$. 


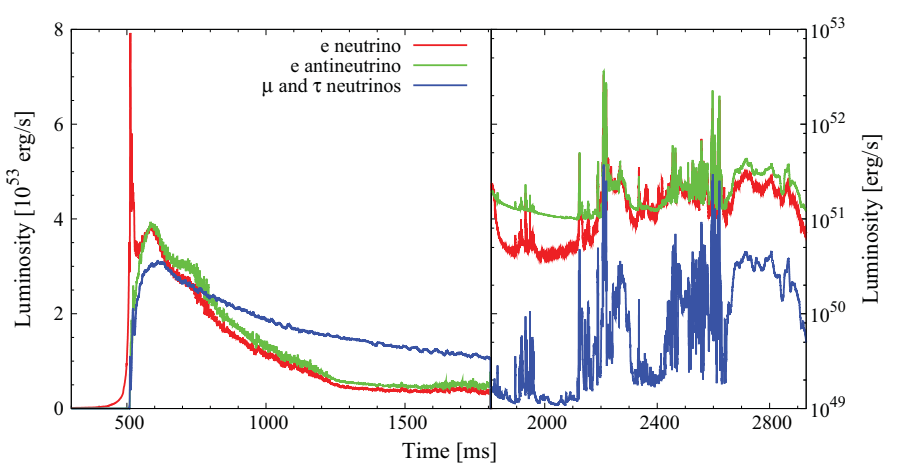

(a)

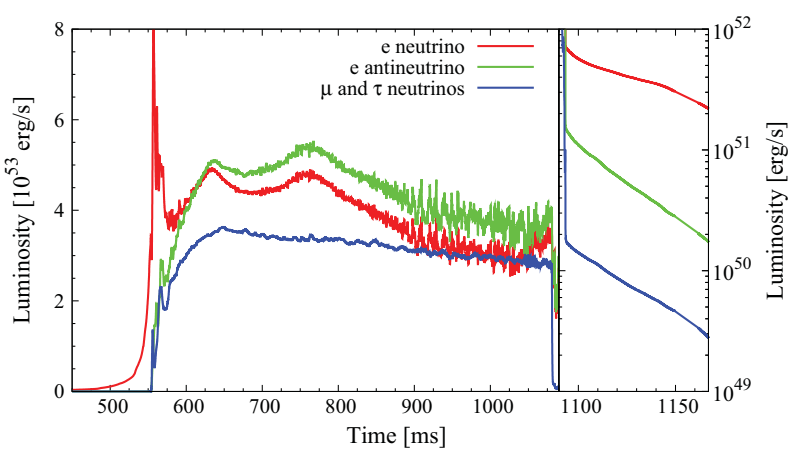

(b)

Figure 2. Time evolution of neutrino luminosities for (a) UN100-rigid and (b) UN100-diff.

characteristic features in UN100-rigid. (ii) At the shock, the kinetic energy associated with the motion perpendicular to the shock surface is dissipated but that associated with the parallel component is preserved. This implies that the infalling material is eventually accumulated in the central region and its kinetic energy is dissipated at the surface of the hypermassive neutron star (HMNS). During this process, oscillations of the HMNS are excited as the infalling matter hits it and the shock waves gain the thermal energy via $P d V$ work and propagate outward. (iii) Due to the accumulation of the matter onto the HMNS and the resulting shock heating, outflows are launched from the polar surface of the HMNS, forming shocks (see Figs. 1(b)). Note that in the present code, neutrino heating is not taken into account and exploring the fate of the thermally driven outflows in the presence of the neutrino heating is an interesting subject. We plan to pursue this issue in the near future. (iv) We found, as another novel feature of dynamics, that the BH-torus system shows time variability (see Figs. 1(c)). Such a time variability has not been seen in UN100-diff (see Figs. 1(d)).

Figures 2(a) and (b) show the time evolution of neutrino luminosities. The neutrino luminosities shows a precipitation when the $\mathrm{BH}$ is formed. The total neutrino luminosity emitted from the HMNS and from the torus around the BH amounts to $L_{\nu \text {,tot }} \sim 10^{53}$ and $\sim 10^{51} \mathrm{ergs} / \mathrm{s}$, respectively. By contrast with the case of UN100-diff, the neutrino luminosities show a violent time variability which is maintained for $\gtrsim 1 \mathrm{~s}$. Such a long-term high luminosity and a time variability may be associated with the time variability that LGRBs show. It is remarkable that the above qualitative differences in dynamics between UN100-diff and UN100-rigid stem from a small difference in the initial angular velocity profile in the outer region (see Fig. 3). Taking into account the dependence of the neutrino pair annihilation rate on the geometry of the torus $\dot{E}_{\nu \bar{\nu}}$ would be given by Beloborodov 


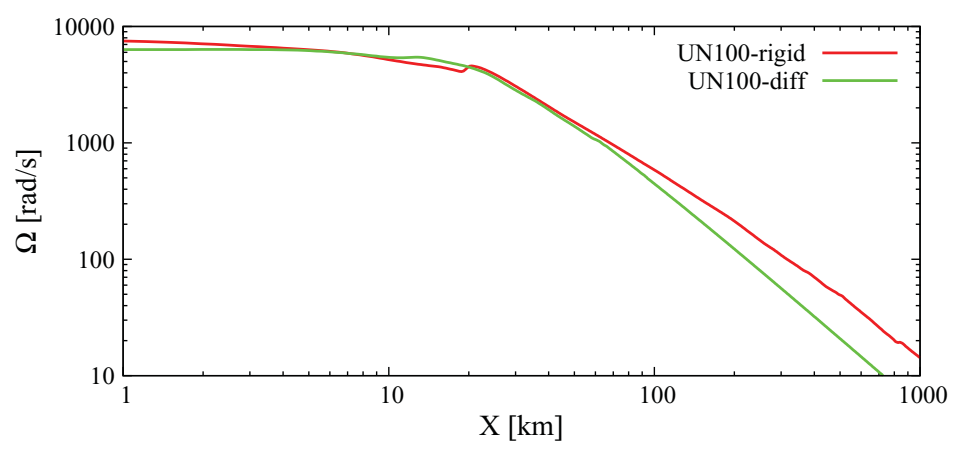

Figure 3. Profiles of the rotational angular velocity along the equator just before the $\mathrm{BH}$ formation for UM100-diff (green curve) and UM100-rigid (red curve).

(2008), $\dot{E}_{\nu \bar{\nu}} \sim 10^{48} \mathrm{ergs} / \mathrm{s}\left(\frac{100 \mathrm{~km}}{R_{\mathrm{fun}}}\right)\left(\frac{0.1}{\theta_{\mathrm{fun}}}\right)^{2}\left(\frac{E_{\nu}+E_{\bar{\nu}}}{10 \mathrm{MeV}}\right)\left(\frac{L_{\nu}}{10^{51} \mathrm{ergs} / \mathrm{s}}\right)\left(\frac{L_{\bar{\nu}}}{10^{51} \mathrm{ergs} / \mathrm{s}}\right) \sin ^{2} \Theta$, where $R_{\text {fun }}$ and $\theta_{\text {fun }}$ are the characteristic radius and the opening angle of the funnel region. $\Theta$ denotes the collision angle of the neutrino pair. Thus a low-luminosity LGRB could be explained. In the HMNS phase, by contrast, the neutrino luminosity is huge as $L_{\nu} \gtrsim 10^{53}$ ergs/s and hence, the deposition rate would be very large as $\dot{E}_{\nu \bar{\nu}} \sim 3 \times 10^{52}$. If the outflows launched due to the mass accumulation mechanism can penetrate the stellar envelope, a system composed of a long-lived HMNS and a geometrically thick torus may be a promising candidate of the central engine of GRBs of relatively short duration.

Future prospects: Recently, we have developed a formulation of general relativistic radiation transfer (Shibata et al. (2011)). We have already performed general relativistic radiation magnetohydrodynamics (GRRMHD) simulations for the evolution of a system composed of a $\mathrm{BH}$ and a surrounding torus with a simplified treatment of microphysics (Shibata \& Sekiguchi (2012)). Furthermore, we have succeeded in implementing a code which can solve the neutrino transfer with a detailed microphysics (in preparation). Using this code, we plan to perform simulations of the stellar core collapse to explore a $\mathrm{SN}$ explosion mechanism and the formation of a $\mathrm{BH}$ in full general relativity.

YS thanks H. Umeda for providing us the presupernova model (UN100) adopted in this work. Numerical simulations were performed on SR16000 at YITP of Kyoto University, on SX9 and XT4 at CfCA of NAOJ, and on the NEC SX-8 at RCNP in Osaka University. This work was supported by Grant-in-Aid for Scientific Research (21018008, 21105511, 23740160) and HPCI Strategic Program of Japanese MEXT.

\section{References}

Beloborodov, A. M. 2008, AIP Conf. Proc. 1054, 51

Fryer, C. L., et al. 2007, PASP, 119, 1211

Sekiguchi, Y. \& Shibata, M. 2005, Phys. Rev. D, 71, 084013

Sekiguchi, Y. \& Shibata, M. 2011, ApJ, 737, 6

Shibata, M. 2003, Phys. Rev. D, 67, 024033

Shibata, M. \& Sekiguchi, Y. 2012, Prog. Theor. Phys., 127, 535

Shibata, M., et al. 2011, Prog. Theor. Phys., 125, 1255

Umeda, H. \& Nomoto, K. 2008, ApJ, 673, 1014

Woosley, S. E. 1993, ApJ, 405, 273

Woosley, S. E. \& Bloom, J. S. 2006, ARAA, 44, 507

Woosley, S. E. \& Heger, A. 2006, ApJ, 637, 914

Zhang, W. \& Woosley, S. E. 2004, ApJ, 608, 365 